\title{
Local one-dimensional reggeon model of the interaction of pomerons and odderons
}

\author{
M. A. Braun, E. M. Kuzminskii, M. I. Vyazovsky ${ }^{\mathrm{a}}$ \\ Department of High Energy Physics, Saint-Petersburg State University, 198504 Saint Petersburg, Russia
}

Received: 12 January 2021 / Accepted: 21 July 2021 / Published online: 31 July 2021

(C) The Author(s) 2021

\begin{abstract}
We propose the one-dimensional reggeon theory describing local pomerons and odderons. It generalizes the well-known one-dimensional theory of pomerons (the Gribov model) and includes only triple interaction vertices. The proposed theory is studied by numerical methods: the oneparticle pomeron and odderon propagators and the pA amplitude are found as functions of rapidity by integrating the evolution equation.
\end{abstract}

\section{Introduction}

In the framework of the Quantum Chromodynamics in the kinematic region where energy is much greater than the transferred momenta ("the Regge kinematics") the strong interactions can be described by the exchange of pomerons, which can be interpreted as bound states of pairs of the so-called reggeized gluons. In the quasiclassical approximation (which neglects pomeron loops) and in the approximation of a large number of colors, for the scattering of a small projectile off a large target ("dilute-dense scattering") it leads to the well-known Balitsky-Kovchegov (BK) equation widely used for the description of DIS and particle-nucleus (pA) scattering. The BK equation corresponds to summing fan diagrams going from the projectile to the nuclear target with the propagator given by the well-known BFKL equation and the triple pomeron vertices responsible for the splitting of a pomeron in two. Going beyond the quasiclassical approximation and taking account of loops present a hardly surmountable problem, which has been not solved until now.

In connection with this difficulty much attention was given to the previous attempts to study the strong interactions before the era of the QCD and using the old reggeon theory [1-3] introduced by V.N. Gribov and based on the phenomenological local pomeron and its interaction vertices. In fact the pA interaction in this framework was consid-

a e-mail: ovsky@MV8660.spb.edu (corresponding author) ered in [4] where the sum of all fan diagrams similar to the BK equation in the QCD was found. Unlike the QCD, in the local pomeron model both the pomeron intercept and coupling constant for the triple pomeron vertex are taken as phenomenological parameters adjusted to the experimental data. Needless to say the local pomeron is much poorer in his physical content as compared to its QCD counterpart, which makes it unfit to describe processes with hard momentum transfer like DIS. However, the local pomeron theory is much simpler than the QCD one and admits various methods which make it possible to go beyond the quasiclassical approximation. In particular renormalization group methods have been widely applied to establish some basic properties of the Gribov model. In [3,5-7] a reggeon field theory with zero renormalized reggeon mass has been investigated by means of the Callan-Symanzik equation and the $\epsilon$-expansion was studied in the vicinity $(D=4-\epsilon)$ of the critical dimension of transverse space. The key result was the existence of an infrared fixed point which leads to scaling laws for the pomeron Green function. More recently a general reggeon field theory was investigated in the framework of the functional renormalization group formalism with the aim of finding fixed points for the coupling constants flows, which may shed light on comparing it with the QCD and relevance for the description of the region of low momenta [8].

Still in the realistic three-dimensional world even the local pomeron model does not allow one to find the full quantummechanical solution of the problem with the contribution of pomeron loops fully taken into account. In view of this trouble a still simpler model ("toy" model) was considered and studied in some detail. The local pomeron model was taken in the one-dimensional world, depending only on rapidity $y$ [913]. Such a model essentially was equivalent to the standard Quantum Mechanics and studied by methods of the quantum mechanics. Later also numerical methods were used both for the evaluation of the eigenvalues of the quantum Hamiltonian $[14,15]$ and for integrating the partial differential equa- 
tion which governs the evolution of this system in rapidity $[16,17]$. The important message which follows from these studies is that the quantum effects, that is, the loops, change cardinally the high-energy behavior of the amplitudes and so the neglect of them is at most a very crude approximation.

It is remarkable that in the QCD, apart from the pomeron with the positive $C$-parity and signature, a compound state of three reggeized gluons with the negative $C$-parity and signature, the odderon, appears. Its possible experimental manifestations has not been found with certainty up to now, which may be explained both by its behavior with energy and its small coupling to participant hadrons. On the theoretical level two species of the odderon were found, the Bartels-LipatovVacca (BLV) odderon [18] with the intercept exactly equal to unity, in which the three reggeized gluons are pairwise located at the same spatial point, and the more complicated Janik-Wosiek odderon $[19,20]$ with all three reggeized gluons at different points, the intercept somewhat below unity and so probably subdominant at high energies. It was noted in [22] that the BLV odderon is in a certain sense an imaginary part of the full $S$-matrix with both $C= \pm 1$ exchanges whose real part is the pomeron. Having this in mind, in analogy with the BK equation a system of equations summing fan diagrams made of both pomerons $P$ and odderons $O$ has been derived [21,22]. It takes into account transitions $P \leftrightarrow P P, P \leftrightarrow O O$ and $O \leftrightarrow P O$ with the coupling constants related as $1:-1: 2$, respectively. This system is more complicated than the BK equation but can be solved numerically as the latter [22-24]. However, going beyond the quasiclassical approximation remains unrealizable. In the framework of the local reggeon model the odderon has been introduced in [25] again within the functional renormalization group formalism and the same goal of finding possible fixed points for the coupling constants flows. However, even forgetting strong limitations on the number of terms in the effective action, the found fixed points tell nothing about the actual values for amplitudes with given phenomenological parameters.

In view of this and learning the lesson from the studies of the theory with only pomerons, in this paper we propose a generalization of the one-dimensional "toy" model to include interactions of both the pomerons and odderons. Note that earlier results found in the functional renormalization group formalism in [25] do not help much to construct such a model due to the essential use of infrared regulators inherent in this formalism. The Lagrangian function of the one-dimensional model with only the pomerons is

$L=\Phi^{+} \partial_{y} \Phi-\mu_{P} \Phi^{+} \Phi+i \lambda \Phi^{+}\left(\Phi+\Phi^{+}\right) \Phi$,

where $\Phi, \Phi^{+}$are the complex pomeron field and its conjugate, the mass parameter $\mu_{P}=\alpha(0)-1$ is defined by the intercept of the pomeron Regge trajectory and $\lambda$ is the effective coupling constant.
To generalize (1) we are guided by the properties of the transition vertices $P \leftrightarrow P P, P \leftrightarrow O O$ and $O \leftrightarrow P O$ found both in the analysis of the loop diagrams in the QCD [26] and in the quasiclassical equations [21,22]. As derived in [26] the one loop corrections should be negative when a pomeron is present in the loop and positive for the odderon loop in the pomeron propagator. From [22] we deduce that the intercept of the odderon is unity and relation of the coupling constants $P \rightarrow P P, P \rightarrow O O$ and $O \rightarrow P O$ should be as in the quasiclassical equations. We have also to take into account the negative signature of the odderon, which makes its contribution to the amplitude real in contrast to the positive imaginary one for the pomeron. As will be discussed in the next section, these properties dictate the form of the Lagrangian in our new local reggeon model to be

$$
\begin{aligned}
L= & \Phi^{+} \partial_{y} \Phi-\mu_{P} \Phi^{+} \Phi+i\left(\Psi^{+} \partial_{y} \Psi-\mu_{O} \Psi^{+} \Psi\right) \\
& +i \lambda\left(\Phi^{+} \Phi^{+} \Phi+\Phi^{+} \Phi \Phi+2 i \Psi^{+} \Psi \Phi+2 i \Phi^{+} \Psi^{+} \Psi\right. \\
& \left.-\Phi^{+} \Psi \Psi-\Psi^{+} \Psi^{+} \Phi\right) .
\end{aligned}
$$

Here $\Phi$ is the complex pomeron field, $\Psi$ is the complex odderon field, $\Phi^{+}$and $\Psi^{+}$are their conjugates, $\mu_{P}$ and $\mu_{O}$ are the pomeron and odderon mass parameters equal to the intercepts minus 1 , respectively, the effective coupling constant $\lambda$ is the same as in the pure pomeron theory (1). To take the odderon signature into account its free Lagrangian is multiplied by $i$, which multiplies its propagator by $-i$. Apart from this we have taken real the coupling constants for $\Psi^{+} \Psi \Phi$ and its conjugate in contrast with all the rest coupling constants kept positive imaginary. This is necessary to conform to the QCD loop contributions and quasiclassical equations $[21,22,26]$. This theory is formally different from the one introduced earlier in [25], however, we shall see that in fact they are fully equivalent after the appropriate transformation of field variables.

This theory describes both creation and absorption of pomerons and odderons and contains interactions only with even powers of the odderon field, hereby conserving the $C$-symmetry. It also has the relation between the coupling constants of different transitions in correspondence with the QCD. In our work we will start with the arbitrary $\mu_{O}$, but in the numerical studies we adopt the zero mass parameter for the odderon in accordance with property of leading BLV odderon.

In the next section from the Lagrangian function (2) we construct the quantum Hamiltonian of the model, study the equations of motion and compare with the quasiclassical (fan) equations in the QCD [21,22]. In Sect. 3 we consider evolution in rapidity of the pomeron and odderon propagators and $\mathrm{pA}$ amplitude in our model and suggest two calculational schemes for its numerical study: development in powers and point-like evolution. In Sect. 4 the numerical results found by these methods are presented. In Sect. 5 the effect of introduc- 
ing of the quartic interaction in the theory is studied. Section 6 contains some conclusions.

\section{The Hamiltonian}

\subsection{Quantization}

The free part of the Lagrangian (2) has the standard form and admit the canonical quantization. Treating $y$ as the imaginary time one finds that the canonical conjugates to the variables $\Phi$ and $\Psi$ are

$\Phi^{*}=\Phi^{+}, \quad \Psi^{*}=i \Psi^{+}$,

respectively. The canonical quantization in the imaginary time leads to the following commutation relations:

$$
\begin{array}{ll}
{\left[\Phi, \Phi^{*}\right]=1,} & {\left[\Psi, \Psi^{*}\right]=1,} \\
{\left[\Phi, \Psi^{*}\right]=0,} & {\left[\Psi, \Phi^{*}\right]=0 .}
\end{array}
$$

The Lagrangian can be presented in the form

$$
\begin{aligned}
L= & \Phi^{*} \partial_{y} \Phi-\mu_{P} \Phi^{*} \Phi+\Psi^{*} \partial_{y} \Psi \\
& -\mu_{O} \Psi^{*} \Psi+V(\Phi, \Psi) .
\end{aligned}
$$

Here $V(\Phi, \Psi)$ is the interaction term which does not contain derivatives in $y$. Then the Euclidean Hamiltonian is

$$
\begin{aligned}
H & =-\left(\Phi^{*} \partial_{y} \Phi+\Psi^{*} \partial_{y} \Psi-L\right) \\
& =-\mu_{P} \Phi^{*} \Phi-\mu_{O} \Psi^{*} \Psi+V(\Phi, \Psi),
\end{aligned}
$$

where the interaction $V$ is

$$
\begin{aligned}
V(\Phi, \Psi)= & i \lambda\left(\Phi^{*} \Phi^{*} \Phi+\Phi^{*} \Phi \Phi+2 \Psi^{*} \Psi \Phi\right. \\
& \left.+2 \Phi^{*} \Psi^{*} \Psi-\Phi^{*} \Psi \Psi+\Psi^{*} \Psi^{*} \Phi\right)
\end{aligned}
$$

Comparing with the pomeron-odderon interaction introduced in [25] we observe that in our case all triple interactions are imaginary but the relative sign of $P \rightarrow O O$ and $O O \rightarrow P$ transitions is opposite. This guarantees that the $O O$ loops have a different sign from $P P$ loops. If one does the canonical phase transformation

$\Psi^{*} \rightarrow \mathrm{e}^{-i \pi / 4} \Psi^{*}, \quad \Psi \rightarrow e^{i \pi / 4} \Psi$

then the two last terms in (7) will appear with the same real coupling constant $\lambda$ as in [25]. So our pomeron-odderon interaction is fully equivalent to one in the latter, provided the three coupling constants there have the same magnitude.

Equations (4) allow one to interpret $\Phi^{*}, \Psi^{*}$ as the creation operators and $\Phi, \Psi$ as the annihilation operators. The vacuum satisfies the condition $\Phi|0\rangle=\Psi|0\rangle=0$ and the Fock space is considered as a full space of states created from the vacuum by action of any number of $\Phi^{*}$ and $\Psi^{*}$. The Schrödinger equation for the wave function of the system $F\left(y, \Phi^{*}, \Psi^{*}\right)$ is

$$
\frac{\partial F\left(y, \Phi^{*}, \Psi^{*}\right)}{\partial y}=-H F\left(y, \Phi^{*}, \Psi^{*}\right) \text {. }
$$

Note that the original field $\Psi^{+}=-i \Psi^{*}$ has a meaning of the creation operator of the odderon state.

2.2 Equations of motion and comparison with the QCD equations for amplitudes

The differential equations of motion for the field operators corresponding to (6) are

$$
\begin{aligned}
\partial \Phi / \partial y= & {[H, \Phi]=\mu_{P} \Phi-i \lambda\left(\Phi^{2}-\Psi^{2}\right) } \\
& -2 i \lambda\left(\Phi^{*} \Phi+\Psi^{*} \Psi\right), \\
\partial \Psi / \partial y= & {[H, \Psi]=\mu_{O} \Psi-2 i \lambda \Phi \Psi-2 i \lambda\left(\Psi^{*} \Phi+\Phi^{*} \Psi\right), } \\
\partial \Phi^{*} / \partial y= & {\left[H, \Phi^{*}\right]=-\mu_{P} \Phi^{*}+2 i \lambda\left(\Phi^{*} \Phi+\Psi^{*} \Psi\right) } \\
& +i \lambda\left(\left(\Phi^{*}\right)^{2}+\left(\Psi^{*}\right)^{2}\right), \\
\partial \Psi^{*} / \partial y= & {\left[H, \Psi^{*}\right]=-\mu_{O} \Psi^{*}+2 i \lambda\left(\Psi^{*} \Phi-\Phi^{*} \Psi\right) } \\
& +2 i \lambda\left(\Phi^{*} \Psi^{*}\right) .
\end{aligned}
$$

Note that the connection (3) with the Hermitian conjugates with respect to the standard scalar product is not conserved during the evolution in $y$ since the Hamiltonian is not Hermitian and the dynamics of the system is not unitary.

We are interested in these equations to compare them in the fan approximation with the QCD. The fan equations are obtained when all terms which contain two creation operators and one annihilation operator are dropped from the Hamiltonian. In our case one has to consider each of Eqs. (9) without the last term in the parentheses. Here we can introduce $\Phi=-i \chi, \Psi=-i \omega$ to get from two first equations

$$
\begin{aligned}
& \frac{\partial \chi}{\partial y}=\mu_{P} \chi+\lambda\left(-\chi^{2}+\omega^{2}\right), \\
& \frac{\partial \omega}{\partial y}=\mu_{O} \omega-2 \lambda \chi \omega .
\end{aligned}
$$

One can see that these fan equations coincide in their form with the fan equations in the QCD.

Indeed, the coupled equations for evolution of the odderon together with the pomeron, derived in [22] in the transverse coordinate space are

$$
\begin{aligned}
& \frac{\partial N(\boldsymbol{x}, \boldsymbol{y} ; y)}{\partial y}=\frac{\bar{\alpha}_{s}}{2 \pi} \int \mathrm{d}^{2} z \frac{(\boldsymbol{x}-\boldsymbol{y})^{2}}{(\boldsymbol{x}-\boldsymbol{z})^{2}(z-\boldsymbol{y})^{2}} \\
& \quad \times[N(\boldsymbol{x}, \boldsymbol{z} ; y)+N(\boldsymbol{z}, \boldsymbol{y} ; y)-N(\boldsymbol{x}, \boldsymbol{y} ; y) \\
& -N(\boldsymbol{x}, \boldsymbol{z} ; y) N(\boldsymbol{z}, \boldsymbol{y} ; y)+O(\boldsymbol{x}, \boldsymbol{z} ; y) O(z, \boldsymbol{y} ; y)], \\
& \frac{\partial O(\boldsymbol{x}, \boldsymbol{y} ; y)}{\partial y}=\frac{\bar{\alpha}}{2 \pi} \int \mathrm{d}^{2} z \frac{(\boldsymbol{x}-\boldsymbol{y})^{2}}{(\boldsymbol{x}-\boldsymbol{z})^{2}(\boldsymbol{z}-\boldsymbol{y})^{2}} \\
& \quad \times[O(\boldsymbol{x}, \boldsymbol{z} ; y)+O(\boldsymbol{z}, \boldsymbol{y} ; y)-O(\boldsymbol{x}, \boldsymbol{y} ; y) \\
& \quad-O(\boldsymbol{x}, \boldsymbol{z} ; y) N(\boldsymbol{z}, \boldsymbol{y} ; y)-N(\boldsymbol{x}, \boldsymbol{z} ; y) O(z, \boldsymbol{y} ; y)]
\end{aligned}
$$


where $N(\boldsymbol{x}, \boldsymbol{y} ; y)$ and $O(\boldsymbol{x}, \boldsymbol{y} ; y)$ are the $C$-even and $C$-odd dipole amplitudes, respectively, symmetric and antisymmetric in $\mathbf{x}, \mathbf{y}$. Here $\bar{\alpha}_{s}=N_{c} \alpha_{s} / \pi$.

Following [23] consider the translational invariant situation when the amplitudes depend only on the difference $\mathbf{r}=\mathbf{x}-\mathbf{y}$. Then passing to the momentum space

$$
\begin{aligned}
& \chi(k, y)=\int \frac{\mathrm{d}^{2} r}{2 \pi r^{2}} N(r, y) \exp (-i k r), \\
& \omega(k, y)=\int \frac{\mathrm{d}^{2} r}{2 \pi r^{2}} O(r, y) \exp (-i k r),
\end{aligned}
$$

we get the equations [23]

$$
\begin{aligned}
& \frac{\partial \chi(k, y)}{\partial y}=-H^{\mathrm{BFKL}} \chi-\bar{\alpha}_{s} \chi^{2}+\bar{\alpha}_{s} \omega^{2}, \\
& \frac{\partial \omega(k, y)}{\partial y}=-H^{\mathrm{BFKL}} \omega-2 \bar{\alpha}_{s} \chi \omega,
\end{aligned}
$$

where $H^{\mathrm{BFKL}}$ is the standard LL BFKL Hamiltonian.

For the local pomeron and odderon both $\chi$ and $\omega$ do not depend on $k$ and depend only on rapidity $y$. Then the equations simplify to

$$
\begin{aligned}
& \frac{\partial \chi(y)}{\partial y}=\mu_{P} \chi(y)-\bar{\alpha}_{s} \chi^{2}(y)+\bar{\alpha}_{s} \omega^{2}(y), \\
& \frac{\partial \omega(y)}{\partial y}=\mu_{O} \omega(y)-2 \bar{\alpha}_{s} \chi(y) \omega(y),
\end{aligned}
$$

where the mass parameters $\mu_{P}$ and $\mu_{O}$ have the meaning of the pomeron and odderon intercepts minus 1 , respectively. If one chooses $\lambda=\bar{\alpha}_{s} \equiv N_{c} \alpha_{s} / \pi$ then Eqs. (15) completely coincide with our Eqs. (10).

It is to be noted that the choice of real vertices for transitions $O \rightarrow O+P$ and $O+P \rightarrow O$ in our original Lagrangian (2) was dictated by the form of the equation for $\partial \omega / \partial y$. With the imaginary vertices one would obtain factor $i$ in the right-hand part, which could not be eliminated by rescaling of $\omega$, since the equation is linear in it.

We acknowledge that the use of the one-dimensional description without the transverse space dependence for the odderon field, which is antisymmetric in $k$, is problematic. The comparison of terms of the equations connected with local interactions can, however, make sense. It shows, at least, that coefficients before different terms and their signs coincide in (9) and (15).

Finally, the comparison of the fan equations fixes the coupling constant for $\Phi^{*} \Psi \Psi$ interaction but not for the last term in (7). Here we choose the constant for the last term in (2) negative imaginary (or, equivalently, the opposite signs before the two last terms in (7)) to take into account the signature properties of odderon. With the given choice of constants the simple loop constructed from two odderon lines gives the positive correction to the pomeron propagator, whereas the simple loop from one odderon line and one pomeron line gives the negative correction both to the pomeron and odderon propagators.

\subsection{Passage to the real Hamiltonian}

The Lagrangian function (2) is to be accompanied by the form of the coupling of the pomerons and odderons to the external particles. We assume the eikonal form for the operators of creation of the initial state and annihilation of the final state (analogous to the ones taken in $[16,17]$ ):

$$
\begin{aligned}
& F^{(\mathrm{in})}=1-\mathrm{e}^{-i g_{P}^{(i)} \Phi^{*}+g_{O}^{i} \Psi^{*}}, \\
& F^{(\mathrm{fin})}=1-\mathrm{e}^{-i g_{P}^{(i)} \Phi-g_{O}^{i} \Psi},
\end{aligned}
$$

with possibly different coupling constants of interaction with the projectile and the target. We choose $g_{p}$ with a negative sign to later deal with well behaved expressions.

The Hamiltonian (6) with interaction (7) is complex, which makes practical use of it inconvenient. So one can pass to a real Hamiltonian. One possibility (case A) to do so is to introduce the Fock-Bargmann representation of operators

$$
\begin{gathered}
u \equiv i \Phi^{*}, \quad v=\frac{\partial}{\partial u} \equiv-i \Phi, \quad w \equiv i \Psi^{*}=-\Psi^{+}, \\
z=\frac{\partial}{\partial w} \equiv-i \Psi .
\end{gathered}
$$

In terms of these operators

$$
\begin{gathered}
H^{(A)}=-\mu_{P} u \frac{\partial}{\partial u}-\mu_{O} w \frac{\partial}{\partial w}+\lambda u^{2} \frac{\partial}{\partial u}-\lambda u \frac{\partial^{2}}{\partial u^{2}} \\
-2 \lambda w \frac{\partial^{2}}{\partial u \partial w}+2 \lambda u w \frac{\partial}{\partial w}+\lambda u \frac{\partial^{2}}{\partial w^{2}}+\lambda w^{2} \frac{\partial}{\partial u} .
\end{gathered}
$$

The initial and final states become

$$
F^{(\text {in })}=1-\mathrm{e}^{-g_{P}^{(i)} u-i g_{O}^{(i)} w}, \quad F^{(\mathrm{fin})}=1-\mathrm{e}^{g_{P}^{(f)} v-i g_{O}^{(f)} z} .
$$

So the coupling of the odderon to the projectile and the target becomes pure imaginary.

Accordingly, our Hamiltonian is now real but the initial and final states are not. To separate real and imaginary amplitudes one has to separate terms with even and odd numbers of odderons, that is, having $C=+1$ and $C=-1$. This can be done separating terms proportional to even or odd powers $m=m_{i}+m_{f}$ of the product $g_{O}^{(i)}{ }^{m_{i}} g_{O}^{(f)^{m_{f}}}$.

The alternative possibility (case B) is to only pass to imaginary $\Phi$ and $\Phi^{*}$ and do not change $\Psi$ and $\Psi^{*}$ :

$$
\begin{gathered}
u \equiv i \Phi^{*}, \quad v=\frac{\partial}{\partial u} \equiv-i \Phi, \quad w \equiv \Psi^{*}=i \Psi^{+}, \\
z=\frac{\partial}{\partial w} \equiv \Psi .
\end{gathered}
$$


and to retain the form of the odderon coupling to the initial and final states. The alternative Hamiltonian is

$$
\begin{aligned}
H^{(B)}= & -\mu_{P} u \frac{\partial}{\partial u}-\mu_{O} w \frac{\partial}{\partial w}+\lambda u^{2} \frac{\partial}{\partial u}-\lambda u \frac{\partial^{2}}{\partial u^{2}} \\
& -2 \lambda w \frac{\partial^{2}}{\partial u \partial w}+2 \lambda u w \frac{\partial}{\partial w}-\lambda u \frac{\partial^{2}}{\partial w^{2}}-\lambda w^{2} \frac{\partial}{\partial u} .
\end{aligned}
$$

The signs of the two last terms will be opposite to the previous case. However, it is trivial to see that this possibility is equivalent to the canonical transformation $\Psi \rightarrow i \Psi, \Psi^{*} \rightarrow-i \Psi^{*}$ and in the end gives the same result for the amplitudes.

\section{Evolution in rapidity}

\subsection{Basic equations and methods for solution}

If the initial state in the Schrödinger picture at rapidity zero is given by

$|y=0\rangle=F_{y=0}\left(\Phi^{*}, \Psi^{*}\right)|0\rangle$,

then at rapidity $y$ it will be given by

$|y\rangle=F_{y}\left(\Phi^{*}, \Psi^{*}\right)|0\rangle$

with the evolution to rapidity $y$ given by the equation

$$
\frac{\partial F_{y}}{\partial y}=-H F_{y}
$$

The amplitude for the transition from the state $F_{y=0}|0\rangle$ to a state $\langle 0| F^{(\text {fin) }}$ at rapidity $y$ will be given by the matrix element

$i \mathcal{A}(y)=\left\langle 0\left|F^{(\mathrm{fin})}(\Phi, \Psi) \mathrm{e}^{-H y} F_{y=0}\left(\Phi^{*}, \Psi^{*}\right)\right| 0\right\rangle$.

We take the Hamiltonian in the real form (18) with the initial and final states in the form (19). The amplitude becomes

$$
\begin{aligned}
i \mathcal{A}(y) & =\left\langle 0\left|\left(1-\mathrm{e}^{g_{P}^{(f)} v-i g_{O}^{(f)} z}\right) \mathrm{e}^{-H y}\left(1-\mathrm{e}^{-g_{P}^{(i)} u-i g_{O}^{(i)} w}\right)\right| 0\right\rangle \\
& =-\left\langle 0\left|\mathrm{e}^{g_{P}^{(f)} \frac{\partial}{\partial u}-i g_{O}^{(f)} \frac{\partial}{\partial w}} F_{y}(u, w)\right| 0\right\rangle
\end{aligned}
$$

where

$$
F_{y}(u, w)=\mathrm{e}^{-H y}\left(1-\mathrm{e}^{-g_{P}^{(i)} u-i g_{O}^{(i)} w}\right)
$$

is the result of the evolution of the initial state to rapidity $y$.

Applying the derivatives we find

$$
i \mathcal{A}(y)=-\left.F_{y}(u, w)\right|_{u=g_{P}^{(f)}, w=-i g_{O}^{(f)}},
$$

the amplitude is obtained by just substituting $u$ and $w$ in the evolved $F(u, w)$ with the coupling constant for the target. To find the propagator one evidently has to take the terms linear in these coupling constants (or in $u$ or $w$ ). So the pomeron and odderon propagators are obtained:

$P(y)=\left.\frac{\partial F(u, w)}{\partial u}\right|_{u=0, w=0}, \quad O(y)=\left.\frac{\partial F(u, w)}{\partial w}\right|_{u=0, w=0}$

with the odderon propagator actually carrying an extra factor $i$.

Passing to the possible methods to solve the evolution equation (24) with the Hamiltonian given by (18) or (21) we can find two basic alternatives. The first one is to study the Hamiltonian and find its eigenvalues. This is a difficult problem already without odderon, as demonstrated by earlier studies. With only the pomeron the Hamiltonian is non-Hermitian and acting on a complex field variable. Fortunately, in this case it could be transformed to a Hermitian Hamiltonian, which allowed one to approximately find its ground state at very small coupling [11]. Later a formalism has been developed to find all eigenvalues from the initial Hamiltonian in the complex variable [15]. Inclusion of the odderon substantially aggravates the situation. Now the initial non-Hermitian Hamiltonian depends on two complex variables. Its analysis requires many more efforts and transformation to a Hermitian Hamiltonian becomes hardly possible.

So we are left with the second alternative, which is to directly integrate the evolution equation numerically. This procedure turned out to be quite efficient for the pure pomeron model at physically reachable energies [16], although of course it cannot be stretched to infinite energies when the groundstate eigenvalue becomes essential. So we turn to direct integration of (24) starting from some initial condition at $y=0$.

One may consider two possibilities for the choice of variables for the wave function. One may develop $F_{y}(u, w)$ in a power expansion in $u$ and $w$,

$F_{y}(u, w)=\sum_{n=0, m=0} g_{n m}(y) u^{n} w^{m}$.

This form has a nice physical interpretation. The term with $u^{n} w^{m}$ corresponds to a state with $n$ pomerons and $m$ odderons. So the expansion (30) immediately gives the pomeronodderon content of the wave function. However, convergence of this expansion is not guaranteed and the cut series obviously has a very bad behavior at large $u$ and $w$. So, as we shall see, the applicability of this method is severely restricted to small values of $y$ and the coupling constant $\lambda$.

Another possibility actually employed in [16] is to take the initial function $F_{y=0}(u, w)$ on the lattice $\left(u_{i}, w_{j}\right)$ and evolve $F$ on this lattice. In this case the evolution equation itself is responsible for the high $y$ and $\lambda$ behavior. As a result this method can be applied in a wide region of $y$ and $\lambda$. 
In the next subsections we consider these numerical methods in more detail.

\subsection{Evolution by power expansion}

Using (30) one finds from (18)

$$
\begin{aligned}
& H F_{y}(u, w)=\sum_{n=0, m=0} g_{n m}(y)\left(-\mu_{P} n u^{n} w^{m}-\mu_{O} m u^{n} w^{m}\right. \\
& +\lambda n u^{n+1} w^{m}-\lambda n(n-1) u^{n-1} w^{m} \\
& -2 \lambda n m u^{n-1} w^{m}+2 \lambda m u^{n+1} w^{m} \\
& \left.+\lambda m(m-1) u^{n+1} w^{m-2}+\lambda n u^{n-1} w^{m+2}\right) .
\end{aligned}
$$

Combining these terms as a coefficient before $u^{n} w^{m}$ one finds

$$
H F_{y}(u, w)=\sum u^{n} w^{m} f_{n m}(y), \quad f_{n m}(y)=H g_{n m}(y),
$$

where at $n \geq 2$ and $m \geq 2$

$$
\begin{aligned}
f_{n m}= & -\mu n g_{n m}-\mu_{O} m g_{n m}+\lambda(n-1) g_{n-1, m} \\
& -\lambda n(n+1) g_{n+1, m}-2 \lambda(n+1) m g_{n+1, m} \\
& +2 \lambda m g_{n-1, m}+\lambda(m+1)(m+2) g_{n-1, m+2} \\
& +\lambda(n+1) g_{n+1, m-2}
\end{aligned}
$$

and for smaller values of $n$ and $m$

$$
\begin{aligned}
f_{00} & =0, \quad f_{10}=-\mu_{P} g_{10}-2 \lambda g_{20}+2 \lambda g_{02}, \\
f_{01} & =-\mu_{O} g_{01}-2 \lambda g_{11}, \\
f_{11} & =-\left(\mu_{P}+\mu_{O}\right) g_{11}-6 \lambda g_{21}+2 \lambda g_{01}+6 \lambda g_{03}, \\
f_{20} & =-2 \mu_{P} g_{20}+\lambda g_{10}-6 \lambda g_{30}+2 \lambda g_{12} \\
f_{21} & =-\left(2 \mu_{p}+\mu_{O}\right) g_{21}+3 \lambda g_{11}-12 \lambda g_{31}+6 \lambda g_{13}, \\
f_{12} & =-\left(\mu_{P}+2 \mu_{O}\right) g_{12}-10 \lambda g_{22} \\
& -4 \lambda g_{02}+12 \lambda g_{04}+2 \lambda g_{20}, \\
f_{02} & =-2 \mu_{O} g_{02}-4 \lambda g_{12}+\lambda g_{10} .
\end{aligned}
$$

Note that if the initial $g_{n m}$ are given at $n, m \leq N$ then one has to require that in (33) terms with $g_{n m}$ outside this domain be put to zero.

The full Hamiltonian describes creation and absorption of pomerons and odderons so that the theory includes loops constructed of both. To study the influence of loops one can consider a reduced fan Hamiltonian which describes fan diagrams and does not contain loops. Denoting the successive eight terms in (18) or (21) as (1), (2), ., (8) one finds that the fan Hamiltonian contains terms $(1)+(2)+(3)+(6)+(8)$ and does not contain the rest.

So in case A the fan Hamiltonian is

$$
\begin{aligned}
H_{\text {fan }}^{(A)}= & -\mu_{P} u \frac{\partial}{\partial u}-\mu_{O} w \frac{\partial}{\partial w}+\lambda\left(u^{2}+w^{2}\right) \frac{\partial}{\partial u} \\
& +2 \lambda u w \frac{\partial}{\partial w}
\end{aligned}
$$

and in $H_{f a n}^{(B)}$ the term with $w^{2}$ will have the opposite sign.

For the fan diagrams in the power representation instead of (33) we find for $n, m \geq 2$

$$
\begin{aligned}
f_{n m}^{f a n}= & -\left(n \mu_{P}+m \mu_{O}\right) g_{n m}+\lambda(n-1+2 m) g_{n-1, m} \\
& +\lambda(n+1) g_{n+1, m-2}
\end{aligned}
$$

and for $n<2$ or $m<2$ with $\mu_{O}=0$

$$
\begin{aligned}
& f_{00}=0, \quad f_{10}=-\mu_{P} g_{10}, \quad f_{01}=0, \\
& f_{11}=-\mu_{P} g_{11}+2 \lambda g_{01}, \\
& f_{20}=-2 \mu_{P} g_{20}+\lambda g_{10}, \quad f_{21}=-2 \mu_{P} g_{21}+3 \lambda g_{11}, \\
& f_{12}=-\mu_{P} g_{12}+4 \lambda g_{02}+2 \lambda g_{20}, \quad f_{02}=+\lambda g_{10} .
\end{aligned}
$$

\subsection{Evolution by points: composite fields}

A method similar to the one used in the previous papers $[16,17]$ is to use the evolution equation as it is, choosing some initial function at a set of points $u_{0}, \ldots, u_{N}, w_{0}, \ldots, w_{N}$ and evolving it with rapidity $y$ by the Runge-Kutta method. As a result of this numerical exercise one gets $F_{y}\left(u_{0}, \ldots u_{N} \mid w_{0}\right.$, $\left.\ldots w_{N}\right)$. This method worked for the pomeron evolution without the odderon in a very wide choice of the model parameters.

Note, however, that this method requires a very particular choice of variables $u$ and $w$. In the Gribov model studied in $[16,17]$ the wave function is an analytic function in $u$. Obviously, any analytical function can be completely determined by its values on some ray in the complex plane. However, one experience drawn from those papers was that the initial condition for the evolution has to be imposed on the positive real axis $i \Phi^{*}=u>0$, otherwise the numerical evolution process breaks down. The reason may be in that in practice one has to limit the values of $\Phi^{*}$ (or $u$ ). Convergence requires the wave function to fall at large values of the argument and this is possible only for a very particular direction in the complex $\Phi^{*}$-plane. As a result one has obligatorily to pass from $\Phi^{*}$ to $u=i \Phi^{*}$ and start the evolution from positive $u$.

With the odderon included we have the same situation with two variables $\Phi^{*}$ and $\Psi^{*}$ and from the start it is not clear which values for these variables in their complex plane allow for convergence. We have immediately discovered that with the choice $u=i \Phi^{*}$ and $w=i \Psi^{*}$ evolution from the positive axes for both variables is impossible, leading to divergence at quite small values of $y<1$.

So we are bound to seek different variables in which evolution becomes realizable. As a hint one may consider a model (which will be the subject of our subsequent paper) in which the fake odderon does not possess the negative signature and moreover has the same intercept as the pomeron, their difference reduced only to $C$-parity. In such a model the choice of variables for evolution is trivial, since it follows this choice for the model with only the pomeron. 
Based on this hint we introduce composite fields as linear combinations of the pomeron and odderon fields. We define

$$
\begin{aligned}
& \varphi=\frac{\Phi+i \Psi}{\sqrt{2}}, \quad \varphi^{*}=\frac{\Phi^{*}-i \Psi^{*}}{\sqrt{2}}, \\
& \tilde{\varphi}=\frac{\Phi-i \Psi}{\sqrt{2}}, \quad \tilde{\varphi}^{*}=\frac{\Phi^{*}+i \Psi^{*}}{\sqrt{2}} .
\end{aligned}
$$

Their commutation relations are

$$
\begin{aligned}
& {\left[\varphi, \varphi^{*}\right]=1, \quad\left[\tilde{\varphi}, \tilde{\varphi}^{*}\right]=1,} \\
& {[\varphi, \tilde{\varphi}]=\left[\varphi, \tilde{\varphi}^{*}\right]=\left[\varphi^{*}, \tilde{\varphi}\right]=\left[\varphi^{*}, \tilde{\varphi}^{*}\right]=0,}
\end{aligned}
$$

which shows that $\varphi$ and $\tilde{\varphi}$ are independent dynamical variables.

If one takes the interaction Hamiltonian as a sum of two interactions of the Gribov model (1) depending on different composite variables and with an appropriate coupling constants one finds

$$
V_{0}=i \sqrt{2} \lambda\left(\varphi^{*} \varphi \varphi+\varphi^{*} \varphi^{*} \varphi\right)+i \sqrt{2} \lambda\left(\tilde{\varphi}^{*} \tilde{\varphi} \tilde{\varphi}+\tilde{\varphi}^{*} \tilde{\varphi}^{*} \tilde{\varphi}\right)
$$

or in terms of elementary fields

$$
\begin{aligned}
V_{0}(\Phi, \Psi)= & +i \lambda\left(\Phi^{*} \Phi \Phi+\Phi^{*} \Phi^{*} \Phi\right. \\
& +2 \Psi^{*} \Psi \Phi+2 \Phi^{*} \Psi^{*} \Psi \\
& \left.-\Phi^{*} \Psi \Psi-\Psi^{*} \Psi^{*} \Phi\right)
\end{aligned}
$$

Comparing with the true interaction $V$ with the odderon signature taken into account we find

$V=V_{0}+V_{1}$

where

$V_{1}=2 i \lambda \Psi^{*} \Psi^{*} \Phi=-i \frac{\lambda}{\sqrt{2}}\left(\varphi^{*}-\tilde{\varphi}^{*}\right)^{2}(\varphi+\tilde{\varphi})$.

So the full Hamiltonian in terms of composite fields is

$H=H_{0}+V_{0}+V_{1}$,

where the free part (in the physical case $\mu_{O}=0$ ) is

$H_{0}=-\frac{1}{2} \mu_{P}\left(\varphi^{*}+\tilde{\varphi}^{*}\right)(\varphi+\tilde{\varphi})$.

Following the experience with only the pomeron field we pass to real variables introducing

$$
\begin{aligned}
\varphi^{*} & =-i u, \quad \varphi=i \frac{\partial}{\partial u}=i v, \quad \tilde{\varphi}^{*}=-i w, \\
\tilde{\varphi} & =\frac{\partial}{\partial w}=i z .
\end{aligned}
$$

We stress that these $u$ and $w$ are not the old $u$ and $w$ in (17). We denote them so only to economize in notation. Then the final Hamiltonian in variables $u$ and $w$ is found as

$$
\begin{aligned}
H= & -\frac{1}{2} \mu_{P}(u+w)\left(\frac{\partial}{\partial u}+\frac{\partial}{\partial w}\right) \\
& -\lambda \sqrt{2}\left(u \frac{\partial^{2}}{\partial u^{2}}-u^{2} \frac{\partial}{\partial u}+w \frac{\partial^{2}}{\partial w^{2}}-w^{2} \frac{\partial}{\partial w}\right) \\
& -\frac{\lambda}{\sqrt{2}}(u-w)^{2}\left(\frac{\partial}{\partial u}+\frac{\partial}{\partial w}\right) .
\end{aligned}
$$

This Hamiltonian is symmetric in variables $u$ and $w$. So once the initial wave function $F(u, w)$ is (anti)symmetric in $u$ and $w$ it will preserve this property in the evolution in rapidity.

It is remarkable that if one takes the initial function $F(u, v)$ on the positive axis of both $u$ and $v$ then its evolution in rapidity becomes possible in a rather wide region of parameters $\mu_{P}$ and $\lambda$ and also to rather high values of the rapidity. The results of this evolution for different sets of $\left(\mu_{P}, \lambda\right)$ are reported in the next section.

Note that the pomeron and odderon fields are expressed as

$$
\Phi^{+}=\Phi^{*}=\frac{\varphi^{*}+\tilde{\varphi}^{*}}{\sqrt{2}}, \quad \Psi^{+}=-i \Psi^{*}=\frac{\varphi^{*}-\tilde{\varphi}^{*}}{\sqrt{2}},
$$

or in terms of $u$ and $w$

$\Phi^{+}=-i \frac{u+w}{\sqrt{2}}, \quad \Psi^{+}=-i \frac{u-w}{\sqrt{2}}$,

and the evolution equation for the wave function takes the form (24) with the Hamiltonian (47). To start evolution in terms of $\varphi, \tilde{\varphi}$ we choose (49), dropping the common factor $(-i)$, as the initial state $F_{y=0}(u, w)$ for the pomeron and odderon, respectively. The expressions for the propagators become

$$
\begin{aligned}
& P(y)=\left.\frac{1}{\sqrt{2}}\left(\frac{\partial}{\partial u}+\frac{\partial}{\partial w}\right) F(u, w)\right|_{u=w=0} \\
& O(y)=-\left.\frac{1}{\sqrt{2}}\left(\frac{\partial}{\partial u}-\frac{\partial}{\partial w}\right) F(u, w)\right|_{u=w=0}
\end{aligned}
$$

instead of (29). Since the exchange of $u$ and $w$ is the $C$ transformation, the $C$-symmetry of the Hamiltonian ensures that the wave function remains symmetrical under $u \leftrightarrow w$ for the pomeron and antisymmetrical for the odderon at any $y$.

Furthermore, $C$-symmetry guarantees that the symmetrical and antisymmetrical parts of the wave function evolve independently, which allows one to choose the sum $u \sqrt{2}$ of (49) as the initial state and to find both propagators from (50) in one numerical simulation. 

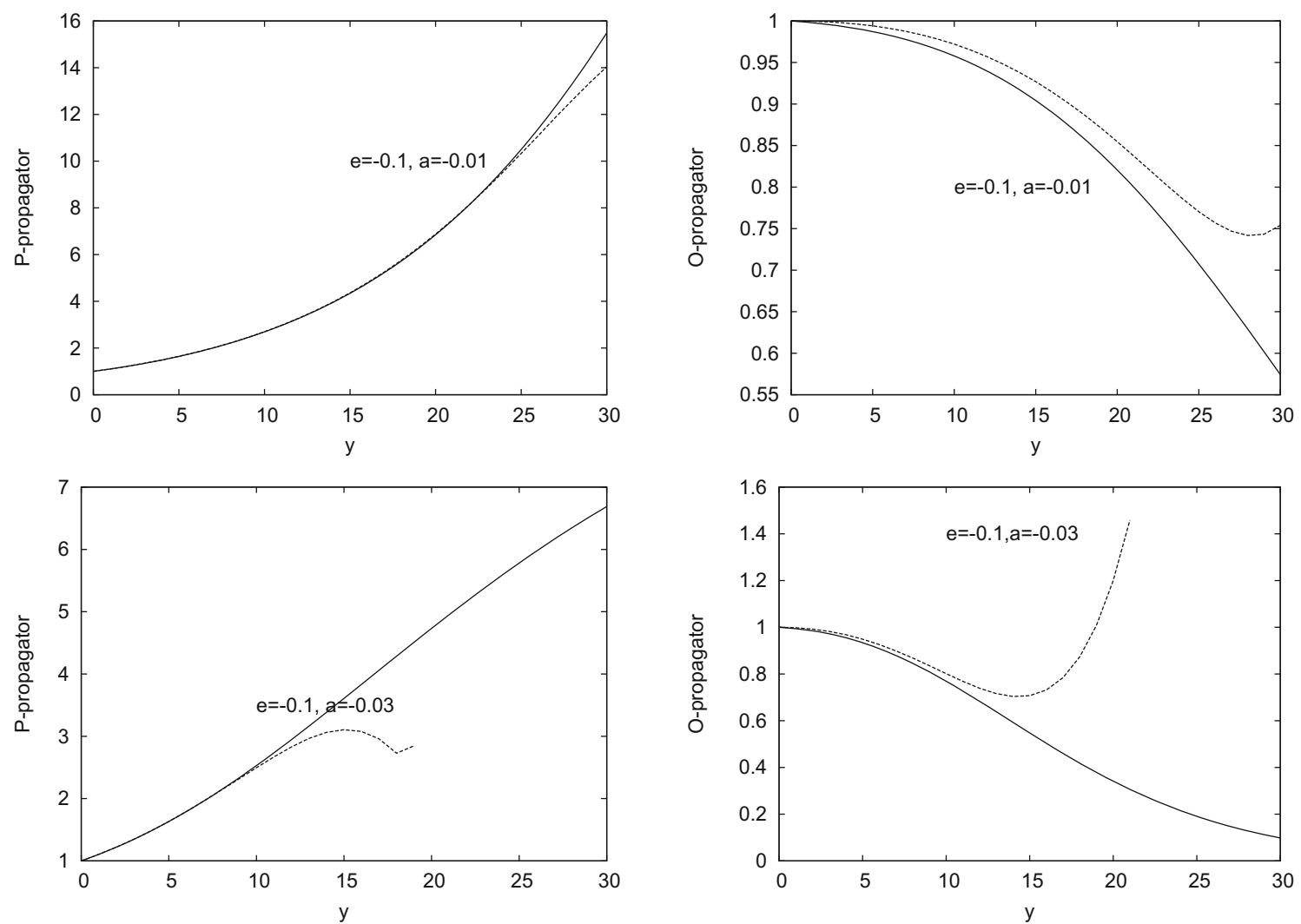

Fig. 1 Propagators of the pomeron (left panels) and odderon (right panels) as calculated by power expansion (dashed curves) and point evolution (solid curves). In all panels $\mu=0.1$. In upper panels $\lambda=0.01$, in the lower $\lambda=0.03$

\section{Numerical evolution: results}

\subsection{Power expansion}

Evolution by power expansion works only for small values of $\mu_{P}$ and $\lambda$. In our calculation we took $\mu=0.1$ and $\lambda=0.01$ and 0.03 . The evolution equations in all cases were solved by the second order Runge-Kutta method with the precision of 10,000 points for $\Delta y=1$. The evolution breaks down at larger $\lambda$. For the chosen $\lambda$ the evolution works approximately until $y=30$ for $\lambda=0.01$ and until $y=20$ for $\lambda=0.03$. The actual interval of $\lambda$ when the evolution works is clear from the figures presenting the results. Note that for technical reasons our figures are labeled by values of $e=-\mu$ and $a=-\lambda$.

The propagators The pomeron propagator $P(y)$ calculated by power expansion for $\mu=0.1$ is shown in Fig. 1 by upper curves in the left upper panel for $\lambda=0.01$ and the left lower panel for $\lambda=0.03$. To compare and see the precision the lower curves present our far more precise results obtained by point evolution. In the right panels we similarly present the odderon propagator for the same values of $\mu$ and $\lambda$. For the odderon the lower curves were obtained by power expansion and the upper ones by point evolution. One observes that the power expansion gives satisfactory results at smaller values of $\lambda$ and rapidity. With the growth of both, its precision worsens notably and at $\lambda=0.03$ its convergence breaks down already at $y \sim 15$.

The influence of loops and the odderon in the whole region $0<y<30$ will be illustrated later, in Fig. 5 based on the precise calculations by the point-like evolution.

The $p A$ amplitude The pA amplitude may be $C$-even and $C$ odd and depend on the couplings of the pomeron and odderon to the proton in the projectile and target. We take $g_{P}^{(i)}=$ $g_{P}^{(f)}=1$ and for the odderon we consider two cases $\left|g_{O}^{(i)}\right|=$ 1 and $g_{O}=0$. In the second case the whole influence of the odderon is reduced to loops. The coupling constant $\lambda$ was again taken as 0.01 and 0.03 .

The $C$-even and $C$-odd amplitudes are presented in Figs. 2 and 3. In Fig. 4 we show the even amplitude with the odderon coupling equal to zero, when the whole influence of the odderon reduces to its loop contribution. In all these pictures the pA amplitude is shown together with the predictions from fan diagrams. As one observes in the $C$-even amplitude the combined effect of the pomeron and odderon loops is the same as in the propagators: they diminish the amplitudes, just as without odderon. The situation with the $C$-odd amplitude is not clear, since it is quite small and the loop effects 

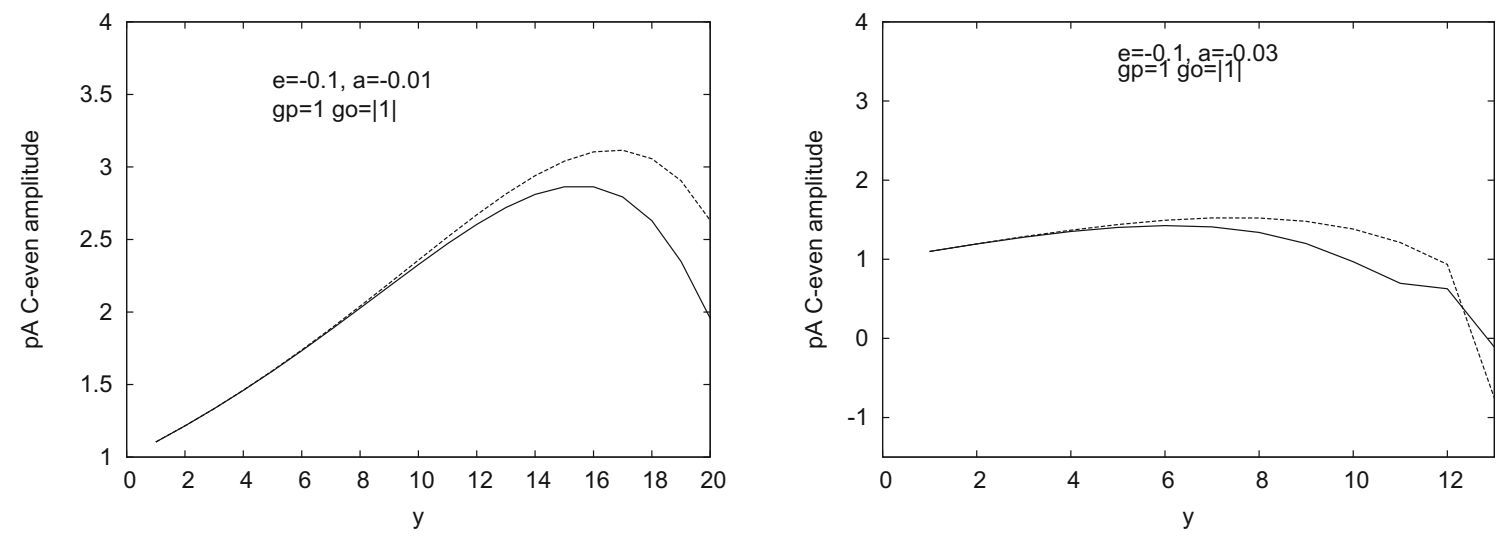

Fig. 2 pA C-even amplitudes for at different rapidities for $\mu_{p}=0.1, \lambda=0.01,0.03$ and $g_{P}=\left|g_{O}\right|=1$. The upper curves correspond to summation of the fan diagrams
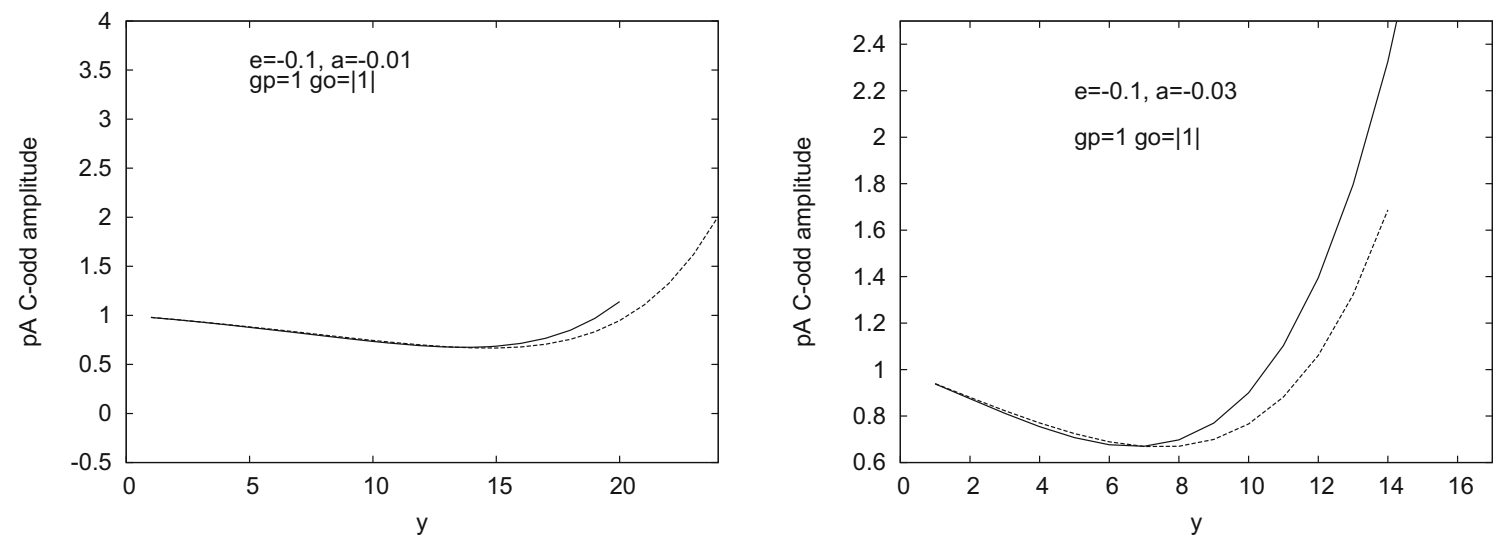

Fig. 3 pA C-odd amplitudes at different rapidities for $\mu_{p}=0.1, \lambda=0.01,0.03$ and $g_{P}=\left|g_{O}\right|=1$. The lower curves correspond to summation of the fan diagrams
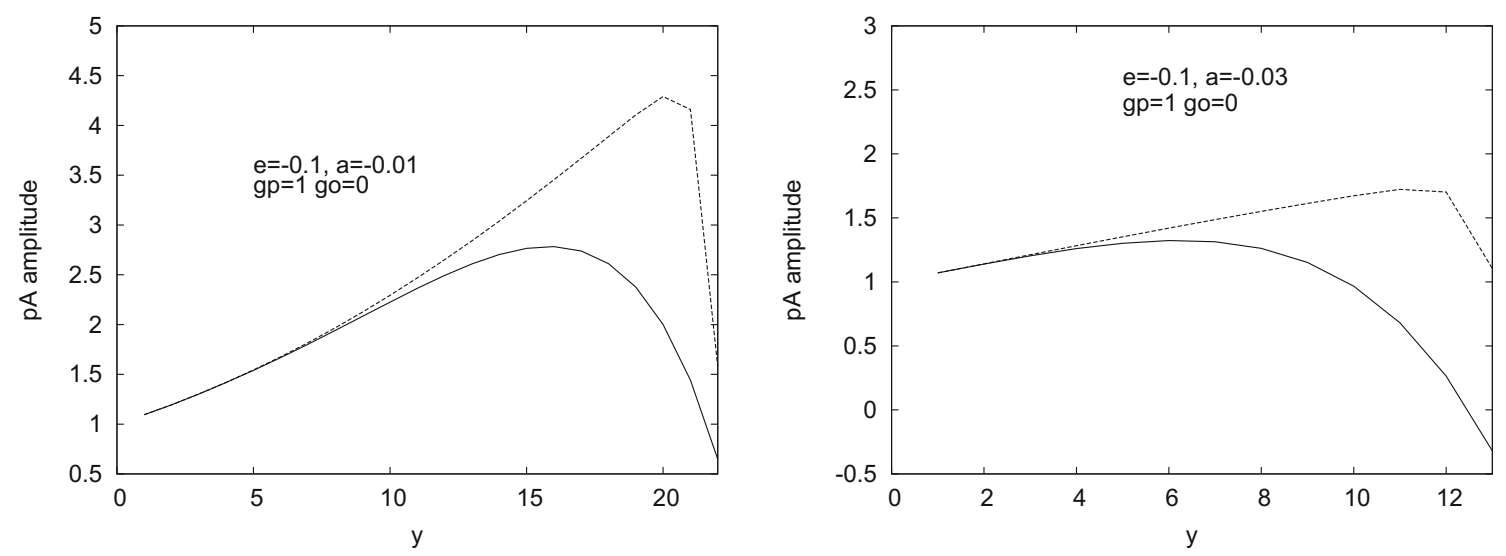

Fig. 4 pA C-even amplitudes at different rapidities for $\mu_{p}=0.1, \lambda=0.01,0.03$ and $g_{P}=1, g_{O}=0$ (lower curves). The upper curves corresponds to summation of the fan diagrams

become visible at rapidities probably outside the region of convergence, which is observed at $y>5$ for $\lambda=0.03$.

\subsection{Evolution by points}

As mentioned, unlike evolution by powers, evolution of Eq. (24) on the $(u, w)$ lattice admits wide areas in $y, \mu$ and $\lambda$ using different steps in $y$. The latter depend on the values 

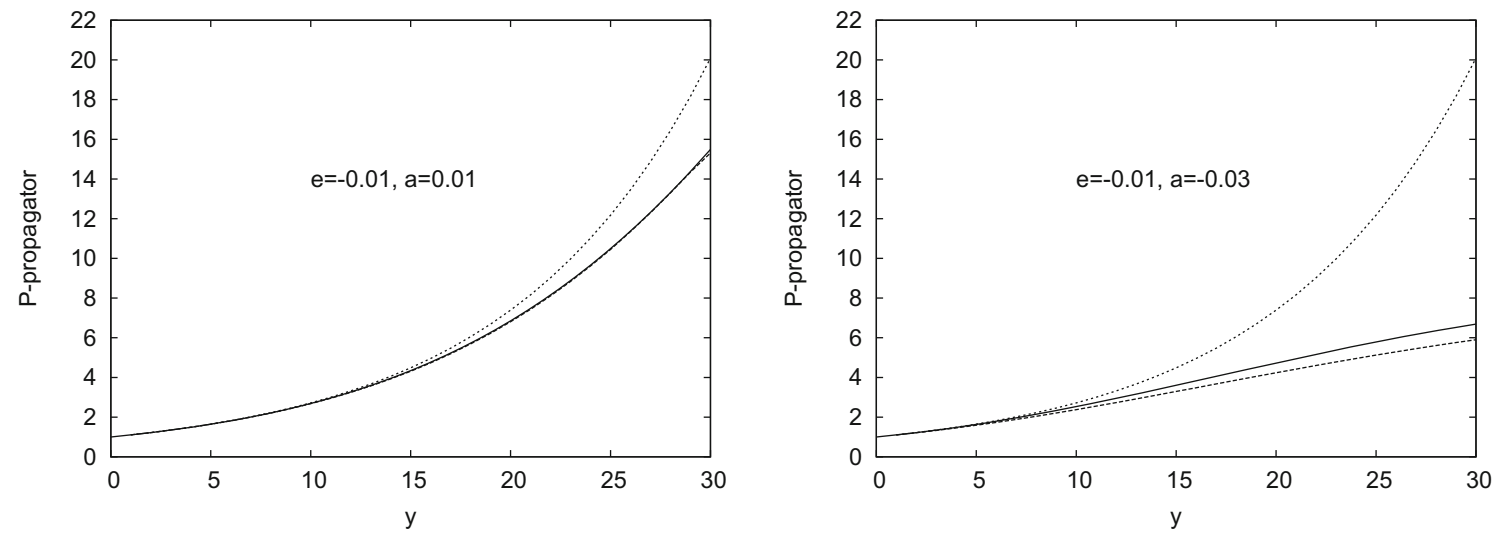

Fig. 5 The pomeron propagators as functions of rapidity for $\mu=0.1, \lambda=0.01$ (left panel) and 0.03 (right panel). Our results with both the pomeron and odderon loops are shown in the middle curve, those with only the pomeron loops in the bottom curve and without loops in the upper curve
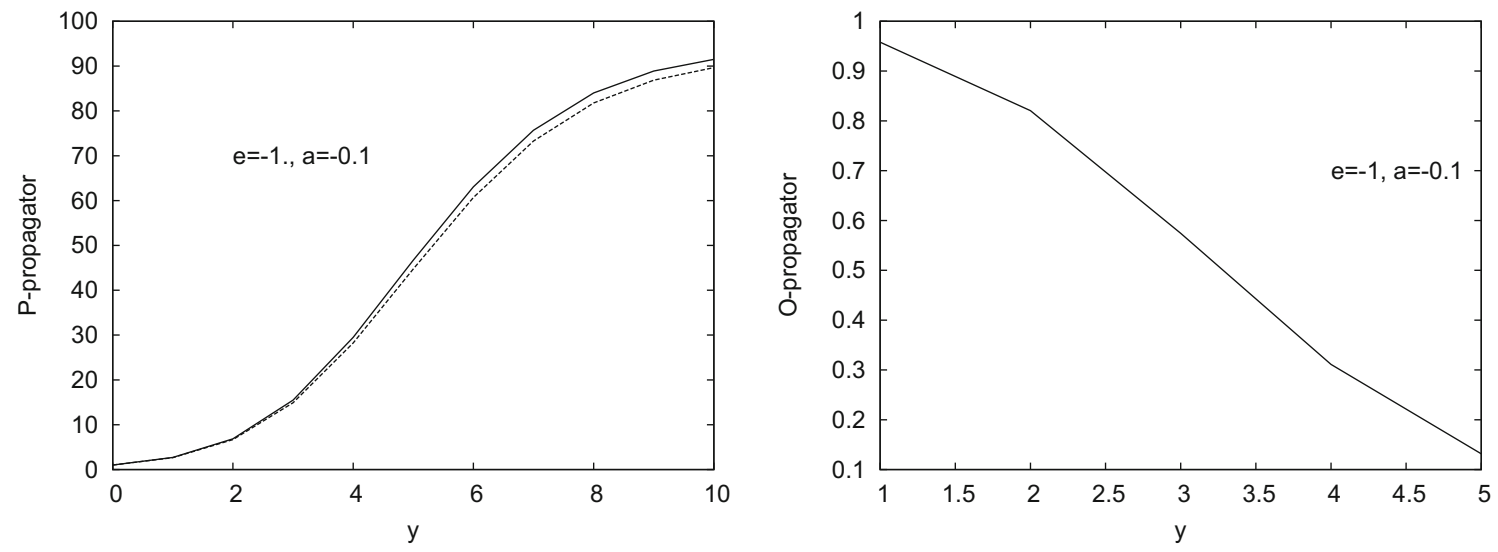

Fig. 6 The solid curves show pomeron (left panel) and odderon (right panel) propagators as functions of rapidity for $\mu=1, \lambda=0.1$. The dashed curve in the left panel shows the pomeron propagator in the absence of the odderon (only pomeron loops)

of $\lambda$. For $\lambda \leq 0.05$ satisfactory results were obtained with $N=400$ points in the interval $(0,20)$ for both $u$ and $w$ and 2000 points for $\Delta y=1$. However, for $\lambda=0.1$ we had to raise the precision in $y$ by taking 20,000 points for $\Delta y=1$. With $\lambda=1$ one has to take 100,000 points for $\Delta y=1$ with the corresponding rise in the processor time. Increasing the number of points in $u$ and $w$ to $N=500$ changes both propagators only by a few percent. Below we report on the calculated pomeron and odderon propagators, for which we took symmetric and antisymmetric initial conditions $(u \pm w) / \sqrt{2}$, respectively. We do not report our results for the pA amplitudes, since their behavior more or less follows the one found above via the evolution by powers.

Pomeron and odderon propagators We start with our results for the pomeron at small values of $\mu$ and $\lambda$, where also power expansion gives converging results previously shown in Fig. 1.

In Fig. 5 the solid (middle) curves show the pomeron propagator with $\mu=0.1$ and $\lambda=0.01$ (left panel) and 0.03 (right panel). The bottom curves show the results obtained with- out the odderon (only pomeron loops) and the upper curves show the propagator without any loops. One concludes that the odderon loops somewhat enhance the propagator, which is natural as they bear the opposite sign.

At $\lambda \geq 0.04$ evolution by power expansion breaks down. So the rest plots for the pomeron and odderon propagators shown in Figs. 6, 7, 8, 9 and 10 use evolution by points. For the pomeron we compared our results including odderon loops with our old calculations without odderon $[15,16]$. We consider the cases with

$(\mu, \lambda)=(1,0.1), \quad(-1,0.1), \quad(1,1 / 3), \quad(1,1), \quad(0.1,1)$,

which fully enough illustrate the dependence on the intercept $1+\mu$ and triple coupling constant $\lambda>0$. The dashed curves in all plots of the pomeron propagator correspond to the results without odderon.

Observing our results we see that the effect of the odderon loops is universally constructive. As expected they act in a direction opposite to the pomeron loops, making the propagator somewhat larger. This effect is enhanced with the 

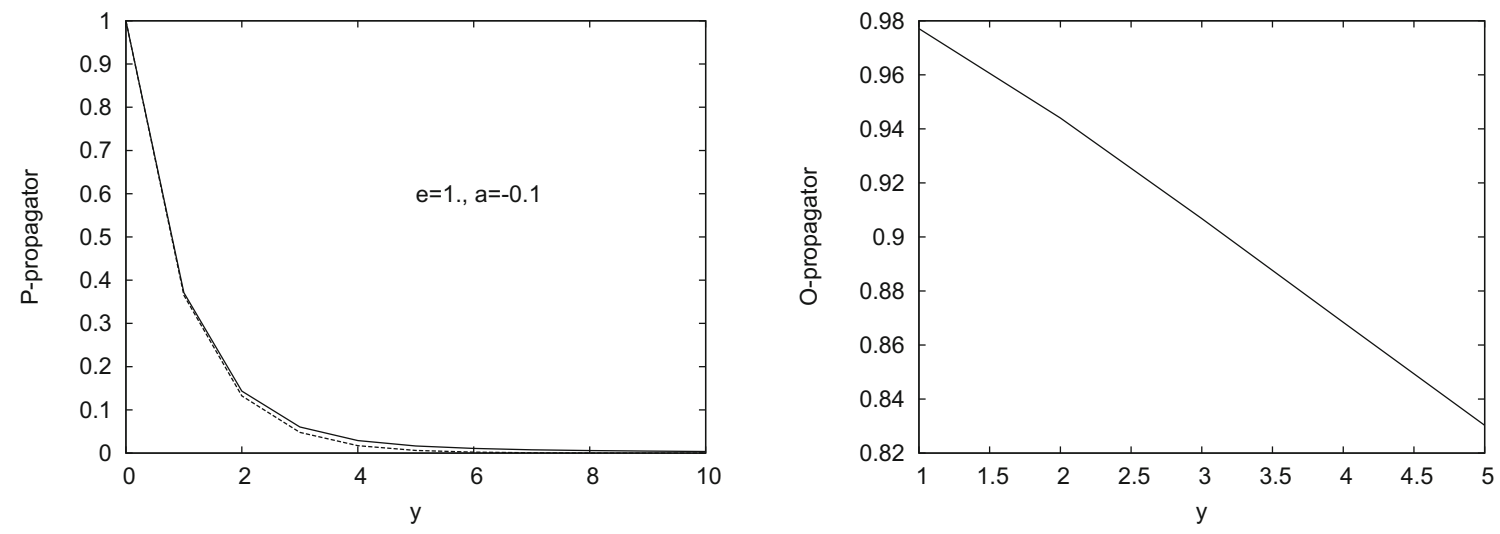

Fig. 7 The solid curves show pomeron (left panel) and odderon (right panel) propagators as functions of rapidity for $\mu=-1, \lambda=0.1$. The dashed curve in the left panel shows the pomeron propagator in absence of the odderon (only pomeron loops)
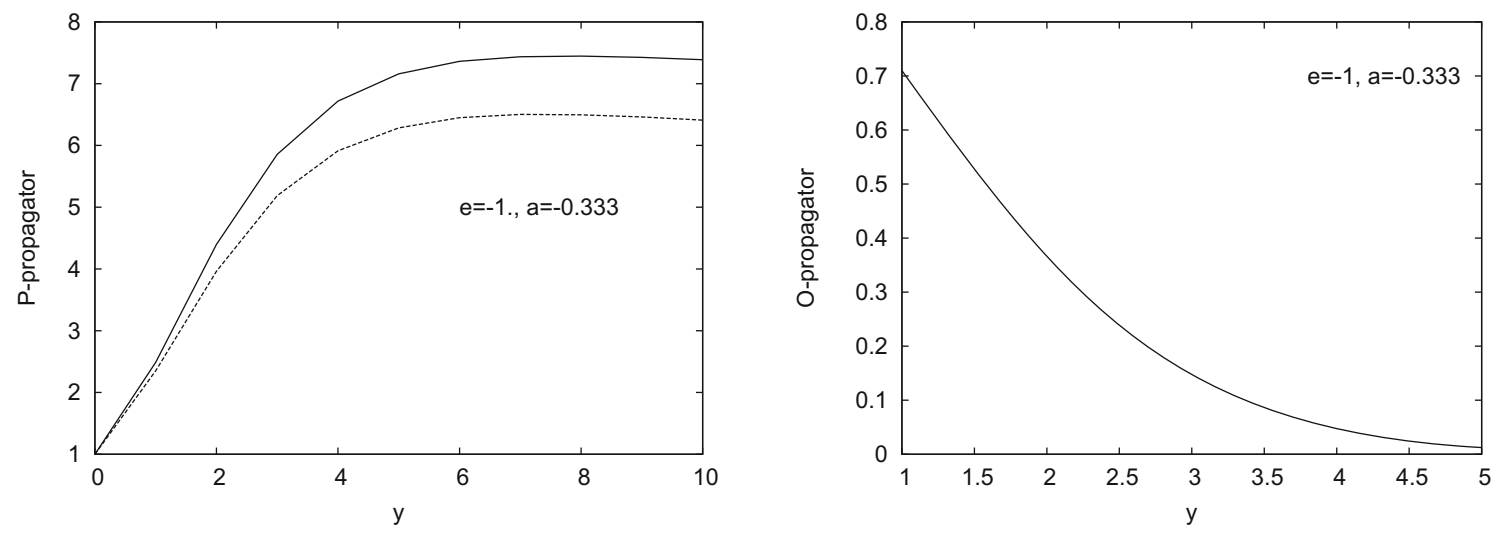

Fig. 8 The solid curves show pomeron (left panel) and odderon (right panel) propagators as functions of rapidity for $\mu=1, \lambda=1 / 3$. The dashed curve in the left panel shows the pomeron propagator in absence of the odderon (only pomeron loops)
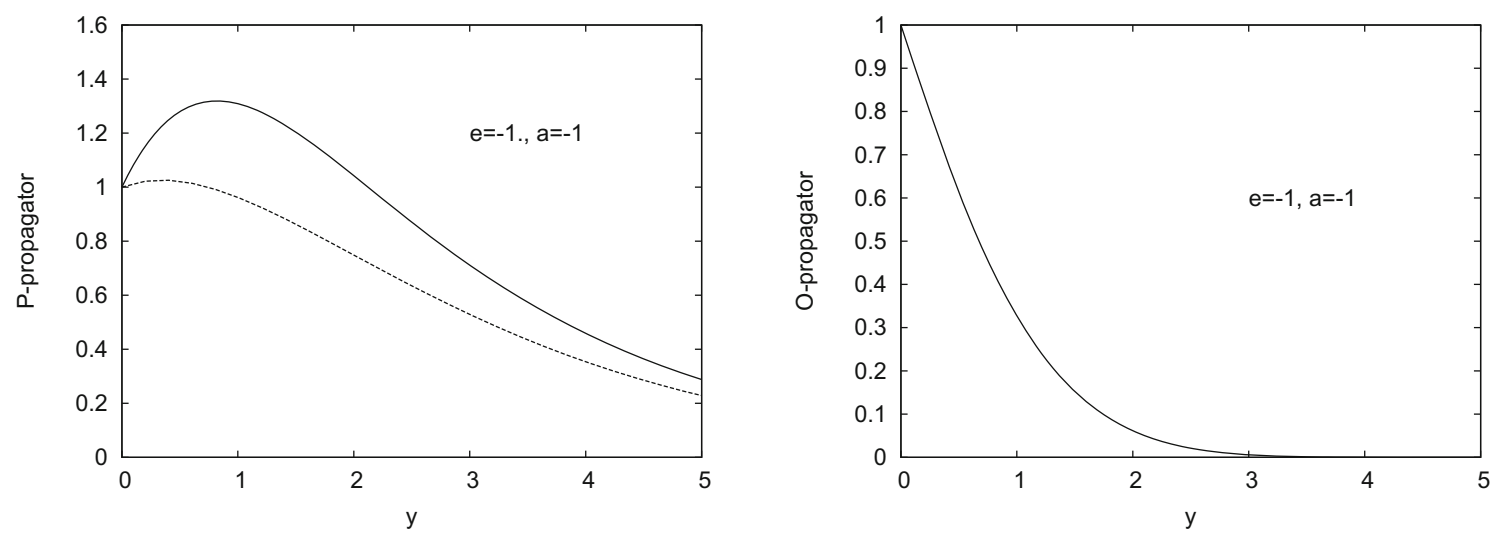

Fig. 9 The solid curves show pomeron (left panel) and odderon (right panel) propagators as functions of rapidity for $\mu=1, \lambda=1$. The dashed curve in the left panel shows the pomeron propagator in absence of the odderon (only pomeron loops)

growth of rapidity. At large values of $\lambda$ the effect of odderon loops starts at quite small $y$ and becomes very strong; see Figs. 8, 9 and 10. In all cases at large enough $y$ the pomeron propagator goes to zero.

\section{Quadruple interaction}

The conclusion that only the triple interactions are essential at large energies is based on the renormalization group analysis $[2,3]$ and is not applicable for the one-dimensional model. To introduce the quadruple interaction in our theory 

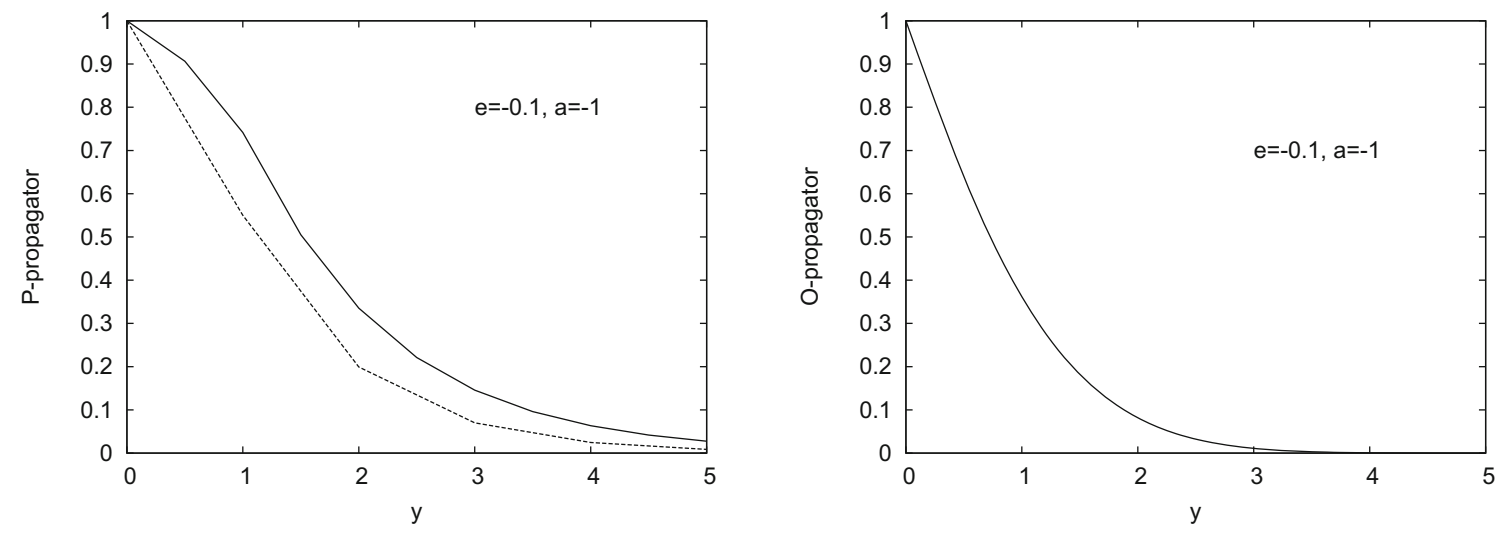

Fig. 10 The solid curves show pomeron (left panel) and odderon (right panel) propagators as functions of rapidity $\mu=0.1, \lambda=1$. The dashed curve in the left panel shows the pomeron propagator in absence of the odderon (only pomeron loops)

we shall exploit the idea that the model can be expressed via the composite fields (38) and add a term to the Hamiltonian

$H_{4}=\lambda^{\prime}\left(\varphi^{*} \varphi^{*} \varphi \varphi+\tilde{\varphi}^{*} \tilde{\varphi}^{*} \tilde{\varphi} \tilde{\varphi}\right)$.

In terms of the pomeron and odderon fields one finds

$$
\begin{aligned}
& \varphi^{*} \varphi^{*} \varphi \varphi=\frac{1}{\sqrt{2}}\left[\left(\Phi^{* 2}-\Psi^{* 2}\right)\left(\Phi^{2}-\Psi^{2}\right)+4 \Phi^{*} \Psi^{*} \Phi \Psi\right. \\
& \left.+2 i\left(\Phi^{* 2}-\Psi^{* 2}\right) \Phi \Psi-2 i \Phi^{*} \Psi^{*}\left(\Phi^{2}-\Psi^{2}\right)\right]
\end{aligned}
$$

Then the quadruple interaction (51) can be written as

$$
\begin{gathered}
H_{4}^{(A)}=\lambda^{\prime}\left(\Phi^{* 2} \Phi^{2}+\Psi^{* 2} \Psi^{2}-\Phi^{* 2} \Psi^{2}\right. \\
\left.-\Psi^{* 2} \Phi^{2}+4 \Phi^{*} \Psi^{*} \Phi \Psi\right) .
\end{gathered}
$$

Passing to variables $u, w(17)$ we have

$H_{4}=\lambda^{\prime}\left(\left(u^{2}-w^{2}\right) \frac{\partial^{2}}{\partial u^{2}}+\left(w^{2}-u^{2}\right) \frac{\partial^{2}}{\partial w^{2}}+4 u w \frac{\partial^{2}}{\partial u \partial w}\right)$.

One can try to use these forms of the interaction to perform the evolution by points in the space $(u, w)$. Unfortunately the additional quadruple terms drastically spoil convergence and do not allow one to move above $y=1$.

To the contrary, a non-zero $\lambda^{\prime}$ evolution by powers allows one to calculate the pomeron and odderon propagators at rather high values of $\lambda$ and $\lambda^{\prime}$ at least up to rapidity $y=10$.

In the evolution in powers of $u$ and $w$ this interaction generates an additional term $f_{n m}^{(4)}$ in (33). For $n, m \geq 2$ one finds

$$
\begin{aligned}
f_{n m}^{(4)}= & \lambda^{\prime}\left\{[n(n-1)+m(m-1)+4 n m] g_{n m}-(m+1)(m+2)\right. \\
& \left.g_{n-2, m+2}-(n+1)(n+2) g_{n+2, m-2}\right\}
\end{aligned}
$$

and for smaller $n$ or $m$

$$
\begin{aligned}
& f_{00}^{(4)}=f_{10}^{(4)}=f_{01}^{(4)}=0, \quad f_{11}^{(4)}=\lambda^{\prime} g_{11} \\
& f_{20}^{(4)}=2 \lambda^{\prime}\left(g_{20}-g_{02}\right), \quad f_{02}^{(4)}=-f_{20}^{(4)} \\
& f_{21}^{(4)}=2 \lambda^{\prime}\left(g_{21}-3 g_{03}\right), \quad f_{12}^{(4)}=2 \lambda^{\prime}\left(g_{12}-3 g_{30}\right) .
\end{aligned}
$$

In Fig. 11 we show the propagators at $\mu=0.1, \lambda=0.1$ and values of $\lambda^{\prime}=0,0.5,1$ and 2 . Convergence becomes better with the growth of $\lambda^{\prime}$. In particular at $\lambda^{\prime}=0$, that is, without the quadruple interaction, the power series method diverges and the corresponding curves could only be obtained by the point evolution.

Calculation shows that the quadruple interaction somewhat raises both the pomeron and odderon propagators which are illustrated in Fig. 11. The curves show the propagators successively enhanced as the values of $\lambda^{\prime}$ are raised: $\lambda^{\prime}=0,0.5,1,2$. So the quadruple interaction (51) acts in the opposite direction to the loops coming from the triple interaction providing a constructive contribution.

\section{Conclusions}

We have proposed a one-dimensional reggeon model for the interaction of local pomerons and odderons at different energies. The model is a natural generalization of the well-known "toy" model for the pomerons only and follows the structure of the pomeron-odderon evolution in the QCD. It allows one to study the evolution of interacting pomerons and odderons with quantum effects (loops) fully taken into account and so estimate these effects and validity of the quasiclassical approximation.

Practical methods to study evolution in rapidity were proposed. In the general case they can be pursued by the same numerical procedure that turned out to be successful for the model without odderons, provided the initial wave function is adequately chosen. In particular, a naive independent 

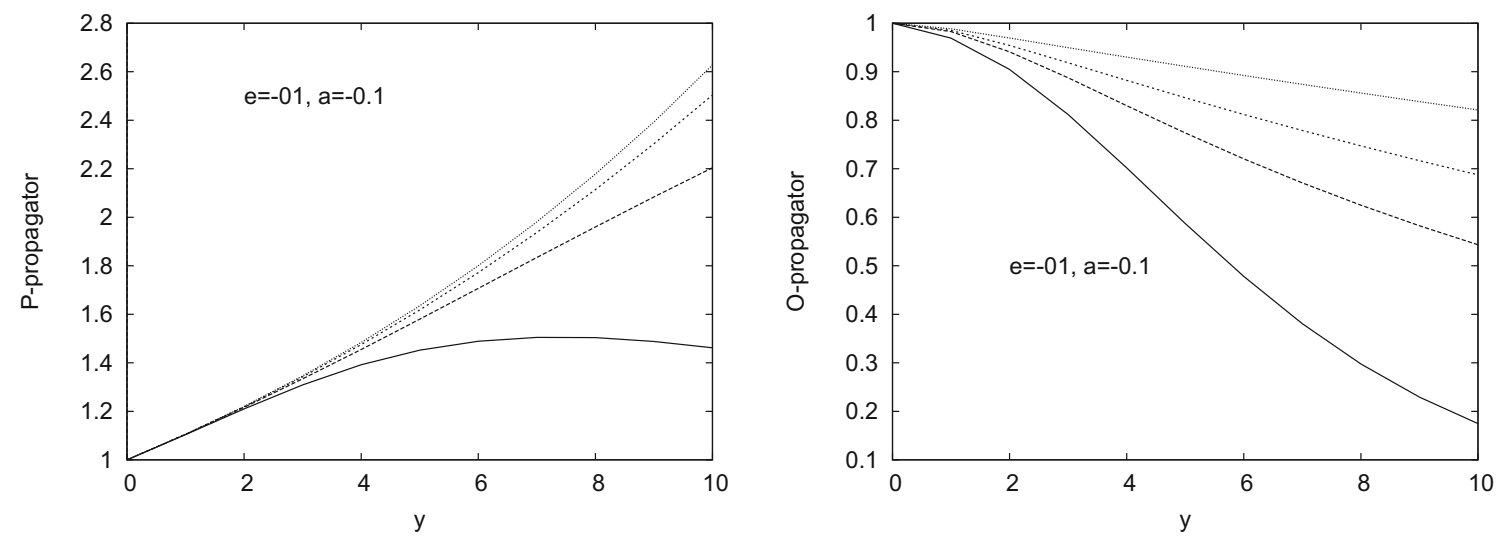

Fig. 11 Pomeron (left panel) and odderon (right panel) propagators at different rapidities for $\mu=0.1, \lambda=0.1$ with the quadruple interaction. Curves from bottom to top correspond to $\lambda^{\prime}=0,0.5,1.0$ and 2.0

initial choice of the pomeron and odderon contribution is not allowed for evolution, since, probably, it contradicts the asymptotic properties of the wave function considered as a function of two complex fields describing the pomeronodderon system.

The numerical results found show that the inclusion of odderon loops enhances the pomeron propagators but somewhat damps the pA amplitudes. In particular, the odderon propagator strongly falls with energy in agreement with conclusions from the QCD. Remarkably the influence of odderon loops is clearly felt both in the propagators and amplitudes. It also leads to overall damping with energies.

We also studied a certain quadruple interaction choosing it in a form symmetric in pomerons and odderons. This interaction acts constructively and raises both the pomeron and odderon propagators.

Acknowledgements The authors are grateful to the referee for major constructive comments and suggestions used in the paper.

Data Availability Statement This manuscript has no associated data or the data will not be deposited. [Authors' comment: All data obtained in the numerical simulations are presented in the plots.]

Open Access This article is licensed under a Creative Commons Attribution 4.0 International License, which permits use, sharing, adaptation, distribution and reproduction in any medium or format, as long as you give appropriate credit to the original author(s) and the source, provide a link to the Creative Commons licence, and indicate if changes were made. The images or other third party material in this article are included in the article's Creative Commons licence, unless indicated otherwise in a credit line to the material. If material is not included in the article's Creative Commons licence and your intended use is not permitted by statutory regulation or exceeds the permitted use, you will need to obtain permission directly from the copyright holder. To view a copy of this licence, visit http://creativecomm ons.org/licenses/by/4.0/.

Funded by SCOAP 3 .

\section{References}

1. V.N. Gribov, Sov. Phys. JETP 26, 414 (1968)

2. A.A. Migdal, A.M. Polyakov, K.A. Ter-Martirosyan, Phys. Lett. 48B, 239 (1974)

3. A.A. Migdal, A.M. Polyakov, K.A. Ter-Martirosyan, Sov. Phys. JETP 40, 420 (1975) [Zh. Eksp. Teor. Fiz. 67, 848 (1974)]

4. A. Schwimmer, Nucl. Phys. B 94, 445 (1975)

5. H.D. Abarbanel, J.B. Bronzan, Phys. Rev. D 9, 2397 (1974)

6. R.L. Sugar, A.R. White, Phys. Rev. D 10, 4074 (1974)

7. A.A. Migdal, A.M. Polyakov, K.A. Ter-Martirosyan, Sov. Phys. JETP 41, 406 (1976) [Zh. Eksp. Teor. Fiz. 68, 817 (1975)]

8. J. Bartels, C. Contreras, G.P. Vacca, J. High Energy Phys. 03, 201 (2016)

9. D. Amati, L. Caneschi, R. Jengo, Nucl. Phys. B 101, 397 (1975)

10. V. Alessandrini, D. Amati, R. Jengo, Nucl. Phys. B 108, 425 (1976)

11. R. Jengo, Nucl. Phys. B 108, 447 (1976)

12. D. Amati, M. Le Bellac, G. Marchesini, M. Ciafaloni, Nucl. Phys. B 112, 107 (1976)

13. M. Ciafaloni, M. Le Bellac, G.C. Rossi, Nucl. Phys. B 130, 388 (1977)

14. S. Bondarenko, Eur. Phys. J. C 71, 1587 (2011)

15. M.A. Braun, E.M. Kuzminskii, A.V. Kozhedub, A.M. Puchkov, M.I. Vyazovsky, Eur. Phys. J. C 79, 664 (2019)

16. M.A. Braun, G.P. Vacca, Eur. Phys. J. C 50, 857 (2007)

17. M.A. Braun, Eur. Phys. J. C 77, 49 (2017)

18. J. Bartels, L.N. Lipatov, G.P. Vacca, Phys. Lett. B 477, 178 (2000)

19. J. Wosiek, R.A. Janik, Phys. Rev. Lett. 79, 2935 (1997)

20. R.A. Janik, J. Wosiek, Phys. Rev. Lett. 82, 1092 (1999)

21. Y.V. Kovchegov, L. Szymanowski, S. Wallon, Phys. Lett. B 586, 267 (2004)

22. Y. Hatta, E. Iancu, K. Itakura, L. McLerran, Nucl. Phys. A 760, $172(2005)$

23. L. Motyka, Phys. Lett. B 637, 185 (2006)

24. M.A. Braun, Phys. Lett. B 809, 135742 (2020)

25. J. Bartels, C. Contreras, G.P. Vacca, Phys. Rev. D 95, 014013 (2017)

26. J. Bartels, M.G. Ryskin, Z. Phys. C 76, 241-255 (1997) 